\title{
Review Article \\ Evaluation of Human Body Fluids for the Diagnosis of Fungal Infections
}

\author{
Parisa Badiee \\ Alborzi Clinical Microbiology Research Center, Namazi Hospital, Shiraz University of Medical Sciences, \\ Zand Avenue, Shiraz 7193711351, Iran \\ Correspondence should be addressed to Parisa Badiee; badieep@yahoo.com
}

Received 18 March 2013; Revised 9 July 2013; Accepted 18 July 2013

Academic Editor: Visith Thongboonkerd

Copyright (C) 2013 Parisa Badiee. This is an open access article distributed under the Creative Commons Attribution License, which permits unrestricted use, distribution, and reproduction in any medium, provided the original work is properly cited.

Invasive fungal infections are a major cause of morbidity and mortality in immunocompromised patients. Because the etiologic agents of these infections are abundant in nature, their isolation from biopsy material or sterile body fluids is needed to document infection. This review evaluates and discusses different human body fluids used to diagnose fungal infections.

\section{Introduction}

Invasive fungal infections (IFI) are increasing in immunocompromised patients due to the improved management of high-risk patients with novel treatment methods. The risk of IFI has been increasing over the last decades mostly because the medical treatments used in specific setting of patients are correlated with severe and prolonged immunosuppression. Although some types of IFI are rare, they are associated with significant morbidity and mortality [1].

The main etiologic agents include yeasts, filamentous fungi, and dimorphic fungi. Common yeasts are predominantly Candida spp. including Candida albicans, Candida glabrata, Candida kefyr, Candida parapsilosis, Candida tropicalis [2], and Cryptococcus spp. Other organisms such as Trichosporon spp. can be involved in serious conditions. The filamentous fungi are classified as having either septate (e.g., Aspergillus spp.) or aseptate hyphae (Mucorales) [3]. Less common pigmented molds (dematiaceous, that is, darkly pigmented) including Pseudallescheria can infect some human organs, especially in the central nervous system (CNS). Finally, the dimorphic fungi are filamentous at $25^{\circ} \mathrm{C}$ and room temperature and yeast-like in host tissues or when incubated at 35 to $37^{\circ} \mathrm{C}$. These fungi, which are true pathogens, include Blastomyces, Histoplasma, Coccidioides,
Paracoccidioides, and Penicillium marneffei and are endemic in specific geographic regions [4-6].

Analysis of all body fluids is essential for the diagnosis of IFI and may be more informative than serum analysis [7]. Invasive diagnostic procedures such as bronchoscopy, thoracoscopy, percutaneous catheter drainage, or open surgery are usually necessary to confirm the diagnosis but are not possible in some critical patients. For pyogenic abscesses, percutaneous catheter drainage is an established technique with a success rate of $80 \%-90 \%[8,9]$, although in deep systemic mycosis of poorly localized nature, the success rate is reported to be low [10].

To date no typical clinical picture has emerged, so the management of infections is critical and the isolation and identification of the fungal pathogen are important for prompt, appropriate therapy. To identify deep-seated IFI and provide appropriate care according to international criteria, isolation of fungi from biopsy specimens [11] or drainage from any focal fluid sample is necessary $[8,9,12]$.

In high-risk patients, the isolation of fungi during followup may appear to contradict the patient's physiological and clinical condition, which may not reflect the severity of the infection. Need for early antifungal therapy is still controversial, especially when Candida is isolated from peritoneal fluid, urine, or pulmonary specimens [13-16]. The isolation of fungi from more than one specimen of urine, peritoneal 
fluid, or blood culture is a reliable criterion for systemic IFI [1].

The methods available for the diagnosis of fungal elements in human body fluids include routine mycological methods such as direct microscopic examination and culture in specific fungal media, serological methods such as the latex antigen test, galactomannan, and mannan antigen or antibody detection by ELISA, and molecular methods such as nested PCR, real-time PCR, and PCR ELISA. Determining the susceptibility of the isolated fungi to antifungal agents can help improve the clinical management of systemic mycosis $[3,17]$. Early diagnosis with prompt antifungal therapy or even surgery might be warranted to save the patient's life [18]. This review evaluates and discusses different human body fluids that can be used in the diagnosis and management of IFI.

\section{Pleural Effusion}

Pulmonary fungal infections are difficult to confirm. The isolation of fungi (especially yeasts) as the pathogenic agent is controversial, so determining the prevalence and management of these infections has been difficult. A previous report demonstrated that Candida colonization could be found in respiratory samples obtained by bronchopulmonary lavage, endotracheal aspirate, or protected specimen brushing in critically ill patients [19]. One of the criteria for the diagnosis of pulmonary FI is an obvious lung lesion on chest X-ray and isolation of fungi from the pleural effusion or blood. The pleural fluid obtained in many cases of fungal pleuritis [20] is a reliable specimen for the diagnosis of pulmonary infections. Pulmonary cryptococcal infection is identified by microscopic examination or positive cryptococcal antigen in percutaneous needle aspiration fluid [18]. For the diagnosis of Aspergillus fungus ball, surgical specimens from the pleural cavity have been used, but the value of pleural fluid is not known [21].

\section{Bronchoalveolar Lavage Fluid}

Pulmonary fungal infection, especially pulmonary aspergillosisis is routinely diagnosed by examination of bronchoalveolar lavage fluid (BAL). There are series of recommendations for performing BAL fluid, according to European Respiratory Society $[22,23]$.

Conventional mycological techniques like culture and histological examination of BAL fluid are the most commonly used ones for the diagnosis of these infections and the detection of the fungal cell wall antigen can be performed by galactomannan (GM) test [24] and PCR [25] in BAL samples. The sensitivity and specificity of galactomannan Ag test can vary in serum and BAL fluid and they are usually lower in BAL than in serum [24]. According to Musher et al., the sensitivity and specificity of the GM EIA in BAL fluid with positive culture result for Aspergillus were $61 \%$ and $98 \%$ with an index cutoff 1.0 and $76 \%$ and $94 \%$ with an index cutoff of 0.5 , respectively [26]. The sensitivity and specificity of qPCR assay in BAL fluid were $67 \%$ and $100 \%$, respectively [26].
As oxygenation is likely to deteriorate during the BAL collection, there are difficulties in performing BAL fluid collection in some critical patients like hematology patients with complication rates approaching $15 \%$ [27] and patients with borderline oxygenation who require elective preprocedure intubation and ventilation.

\section{Peritoneal Fluid}

For some patients a permanent vascular access cannot be used, and peritoneal dialysis is performed instead. In such patients, fungal peritonitis [28] is one of the most serious complications. This infection is a rare but potentially fatal complication. Fungal peritonitis accounted for 3.6\% [29] and from $5 \%$ to $22 \%[30,31]$ of all peritonitis episodes. The mortality rate was from $20 \%$ to $30 \%$ in one study [32] and $60 \%$ to $70 \%$ in another [33]. However, in some areas the rates can be much higher, and peritonitis is associated with high rates of morbidity and mortality.

Direct microscopic examination of the peritoneal fluid was useful for confirming suspected IFI in $60 \%$ of patients in one study [29]. In patients in the intensive care unit with peritonitis, grade $\mathrm{C}$ scores (at least three risk factors) predicted yeast isolation from peritoneal fluid with $84 \%$ sensitivity, $50 \%$ specificity, $67 \%$ positive predictive value, $72 \%$ negative predictive value, and an overall accuracy of $71 \%$ [34]. About $43.4 \%$ of the patients with perforated peptic ulcer [35] had positive peritoneal fluid fungal culture for yeast, including Candida spp. The agents responsible for peritonitis according to Martos et al. were Candida parapsilosis (4), $C$. albicans (2), C. tropicalis (1), C. glabrata (1), C. famata (1) and Fusarium oxysporum (1) [29].

\section{Urine}

Candiduria is clearly not a disease and is common in hospitalized patients. Clinical findings vary and can include asymptomatic candiduria (previously healthy patients, predisposed outpatients, or predisposed inpatients), symptomatic candiduria (cystitis, pyelonephritis, prostatitis, epididymoorchitis, or urinary tract fungus balls), and clinically unstable candiduria [36]. Most patients are asymptomatic. C. albicans is the yeast most commonly isolated from urine, accounting for $50 \%$ to $70 \%$ of the isolates in various studies [37-39].

Samples should be collected with the clean-voided urine culture method. Candiduria may occur due to contamination of urine during sampling with perineal flora, especially in older women or when vaginal discharge is present. In these cases, sampling must be repeated and it is often necessary to obtain the urine specimen by sterile bladder catheterization. If the second specimen culture yields no yeasts, contamination can be assumed and no further diagnostic procedures are needed.

Once contamination is ruled out, colonization of the bladder, perineum, or indwelling urinary catheter must be considered. To verify infection from colonization, the number of organisms in the urine must be quantified. The first studies done in 1970s used renal biopsies to establish renal 
involvement $[31,40]$. In patients without a catheter or with a short indwelling catheter, proven renal infection was found with as few as 10000 to 15000 yeasts $/ \mathrm{mL}$ and as many as 40000 yeasts $/ \mathrm{mL}$ in the urine [41]. In addition, criteria such as pyuria, the presence of pseudohyphae [42], and the finding of casts $[43,44]$ in the urine were considered to distinguish between colonization and urinary tract infection, but these features are of limited practical use in clinical terms. Species such as Candida glabrata naturally cannot produce pseudohyphae, and C. albicans can be induced to form pseudohyphae by varying $\mathrm{pH}$ and nutrient conditions. It was also thought that antibody-coated yeasts in the urine could be used as a marker for infection $[45,46]$.

Some special disorders such as acquired or congenital disturbances of urine flow, occult diabetes mellitus, genitourinary structural abnormalities, bacterial infections, diminished renal function, structural abnormalities of the kidney, and metabolic abnormalities merit particular attention because they are the predisposing factors for candiduria [47, 48]. Candiduria may be due to hematogenous seeding of the kidney cortex in the course of disseminated candidiasis [37]. Experimental studies of hematogenous renal candidiasis in animal models indicated that any concentration of Candida spp. in the urine was significant for renal involvement [49]. Even if infection of the urinary tract by Candida spp. can be confirmed, surprisingly, physicians do not always follow the patients and antifungal therapy is not always warranted [3739]. This issue needs to be addressed in further studies of the patients with candiduria.

\section{Pericardial Effusion}

Fungal pericarditis occurs mainly in immunocompromised patients [50] due to endemic fungi such as Histoplasma and Coccidioides, opportunistic fungi (Candida, Aspergillus), and semifungi including Nocardia and Actinomyces [51-53]. The clinical picture of fungal myocarditis comprises of the full spectrum of pericardial diseases [54]. Fungal pericarditis is diagnosed mainly by staining and culturing pericardial effusion or tissue samples. The samples must be analyzed promptly and should undergo Gram, acid-fast, and fungal staining, followed by cultures [55]. Molecular assays can serve as a reliable method for the diagnosis of fungal endocarditis [56].

\section{Blood}

Diagnostic methods for IFI in the blood vary in sensitivity and specificity. Blood culture for the diagnosis of filamentous fungi is not specific and for Candida spp., with a sensitivity about 50\% [57, 58]. Candidemia is diagnosed when Candida spp. are isolated from at least one blood culture [59]. The mortality rates for candidemia range from $5 \%$ in intensive care units in the USA to $71 \%$ in liver transplant recipients [60].

Serological methods such as the beta glucan assay in serum may be useful for identifying IFI by all etiologic agents except Cryptococcus and Zygomycetes, with sensitivity rates of $64 \%$ to $77 \%$, although specificity may be decreased in patients with certain concurrent bacterial infections [61, 62]. The serum galactomannan assay was found to have $95 \%$ sensitivity and specificity for invasive Aspergillus infections [63], and the serum mannan assay had a $67 \%$ specificity and $90 \%$ sensitivity for the diagnosis of systemic candidiasis in patients with fungemia $[64,65]$.

Molecular methods have recently been used to detect systemic IFI in serum and whole blood samples. The panfungal PCR assay [61] detects the small-subunit rRNA gene sequence of major fungal organism groups, with a sensitivity of $80 \%$ and a specificity of $95.6 \%$ [66]. Other molecular methods include PCR ELISA, nested and real-time PCR, which are used to detect fungal DNA in blood samples. These methods can improve the monitoring of the patients after antifungal therapy $[67,68]$. In patients with IFI who respond to antifungal therapy, PCR ELISA assays became negative after 14 days of treatment and remained positive in patients who did not respond [69]. The other advantage of molecular methods for blood samples, especially in serial sampling, is the earlier appearance of positive PCR findings compared to other diagnostic methods based on radiological symptoms. Culture methods can take 8-10 days, whereas PCR can yield results in 4-5 hours. In patients who were followed weekly, the PCR findings became positive before clinical manifestations and radiological findings appeared. In other words, the PCR results became positive during the incubation period of the infection $[66,70,71]$.

\section{Cerebrospinal Fluid}

Many fungi can cause CNS infection in high-risk patients. Infection in immunocompetent patients has also been reported [72]. Among patients with hematologic malignancies, CNS disease accounts for $9.4 \%$ of filamentous IFI [73]. The diagnosis is based on the analysis of cerebrospinal fluid (CSF). In adult normal CSF, the white blood cell (WBC) count may be as high as $5 / \mathrm{mL}$ with a predominance of lymphocytes. In normal CSF the glucose concentration is from $50 \%$ to $60 \%$ of serum values [74].

In patients with fungal meningitis infection, the WBC count is variable and lymphocytic pleocytosis (Cryptococcus, Candida) is present together with a predominance of neutrophils (Aspergillus, Blastomycosis) or eosinophilia (Coccidioides) [75].

On direct microscopic examination, hyphae or pseudohyphae can occasionally be seen in Candida or other infections. Indian ink should be used for the diagnosis of Cryptococcus neoformans. Fungal cultures are mostly positive in $C$. neoformans and candidal meningitis and are less frequently positive in other IFI [76]. Larger volumes of CSF obtained with repeated lumbar puncture can increase the chances of positive culture results.

Glucose and protein concentrations in CSF are the sensitive indicators of CNS pathology. The physician should know the normal reference range in each region because the measurements are technique dependent and the normal range varies depending on laboratory procedures. In the normal population, CSF glucose is about two thirds of the 
TABLE 1: Use of body fluid for the detection of fungal infections.

\begin{tabular}{|c|c|c|}
\hline Site of infection & Sample & Most Etiologic fungal agents \\
\hline Systemic infection & Blood & Candida spp., Aspergillus spp., Cryptococcus spp. \\
\hline $\begin{array}{l}\text { Urinary tract and systemic } \\
\text { infection }\end{array}$ & Urine & $\begin{array}{l}\text { Candida spp. especially C. albicans, Cryptococcus } \\
\text { spp. }\end{array}$ \\
\hline 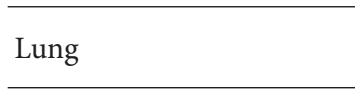 & $\begin{array}{l}\text { Pleural effusion, Bronchoalveolar } \\
\text { lavage fluid }\end{array}$ & $\begin{array}{l}\text { Candida spp., Aspergillus spp., Cryptococcus spp., } \\
\text { Zycomycetes fungi family }\end{array}$ \\
\hline Peritoneum & Peritoneal & $\begin{array}{l}\text { Candida spp., and rare filamentous fungi like } \\
\text { Fusarium oxysporum or Aspergillus spp. }\end{array}$ \\
\hline Joint & Synovial fluid & Candida spp., Aspergillus fumigatus \\
\hline Heart & Pericardial effusion & $\begin{array}{l}\text { Endemic fungi such as Histoplasma and } \\
\text { Coccidioides or opportunistic fungi like Candida, } \\
\text { Aspergillus and semi-fungi including Nocardia } \\
\text { and Actinomyces }\end{array}$ \\
\hline Central Nervous Infection & Cerebrospinal fluid & $\begin{array}{l}\text { Cryptococcus neoformans, Candida spp., } \\
\text { Aspergillus spp., Blastomyces dermatitis, } \\
\text { Pseudallescheria and Histoplasma capsolatum }\end{array}$ \\
\hline Oral & Saliva & Candida spp. \\
\hline
\end{tabular}

TABLE 2: Diagnostic methods for the detection of opportunistic fungal infections in body fluids.

\begin{tabular}{ll}
\hline Etiologic agents & Diagnostic methods \\
\hline & Pleural effusion or Bronchoalveolar lavage fluid \\
& (i) Direct examination, cultures, histopathologic demonstration \\
& (ii) Galactomannan antigen detection, Beta-D-glucan assay*, Polymerase chain reaction \\
& Serum \\
Aspergillosis & (i) Aspergillus antibody test (precipitins) for chronic pulmonary aspergillosis \\
& (ii) Aspergillus IgG antibodies, Aspergillus IgE test (precipitins) \\
& (iii) Galactomannan and beta-D-glucan levels double sandwich enzyme-linked immunosorbent \\
assay (ELISA) \\
(iv) 1,3-Beta-D-glucan
\end{tabular}

Blood, CSF, Urine, or other body fluid

Candidiasis
(i) Isolating a Candida species from multiple or repeat cultures
(ii) Beta-D-glucan and other antigen and metabolite assays
(iii) Polymerase chain reaction

Cerebrospinal fluid, Blood or Serum, BAL, Urine

\begin{tabular}{ll} 
Cryptococosis & (i) India ink smear \\
& (ii) Culture \\
& (iii) latex agglutination test or enzyme linked immunosorbent for Cryptococcal antigen testing \\
\hline
\end{tabular}

Mucormycosis Sterile body fluid from site of infection

(i) Histopathopathologic examination

(ii) Culture

Other fungal infection Sterile body fluid from site of infection

(i) Histopathopathologic examination

(ii) Culture and isolation of fungal species in cultures of involved biologic materials

$\bar{*}$ 1,3-Beta-D-glucan, a cell wall composition of many fungi, may be positive in patients with a variety of invasive fungal infections, it is typically negative in patients with mucormycosis or cryptococcosis.

serum glucose concentration and does not exceed $300 \mathrm{mg} / \mathrm{dL}$ regardless of serum levels. The adult range of CSF protein concentration reaches 18 to $58 \mathrm{mg} / \mathrm{dL}$ between 6 and 12 months of age [77]. In fungal brain infections, CSF glucose is generally low and protein is generally high, with exceptionally high levels in cryptococcal infections [74].

The sensitivity and specificity rates for CSF antigen testing are above $90 \%$ for Cryptococcus and Histoplasma [74, 75] and 
the galactomannan CSF assay for the diagnosis of invasive aspergillosis has been investigated [63]. In addition, PCR analyses can indicate the presence of DNA from different fungal species in CSF [78].

\section{Synovial Fluid}

Fungal joint infection due to yeast and filamentous fungi is rare and its diagnosis and treatment can be challenging even for skilled doctors. The etiologic agents reported most frequently are Candida and Aspergillus spp. In invasive Aspergillus infections, dark fluid is aspirated from the joint. In joints with Aspergillus fumigatus infection, synovial fluid analysis showed cell count between 7300 and $128000 \mathrm{cell} / \mathrm{mL}$, predominantly neutrophils [79].

In recent years the incidence of periprosthetic joint infection (total knee replacement) has increased. In one study, most fungal knee infections were caused by Candida species (80\%), of which C. parapsilosis was the most common (50\%) [80]. The definitive diagnosis and identification of the etiologic agent were yielded by aspiration of synovial fluid in most cases, but in some cases intraoperative and tissue biopsy culture were needed [81].

\section{Saliva}

Saliva is the first defense against the many pathogenic organisms in oral cavity. With regard to IFI, the most important role of saliva is defense against pathogenic fungi with special enzymes. Candida albicans is the most important fungal species in the oral cavity. The primary mechanism of salivary defense is secretory immunoglobulin, which inactivates fungi via binding and/or agglutination of the microorganisms [82]. Other defense mechanisms have also been hypothesized, such as lactoferrin, lysozyme, and histamine, particularly histatin-5, which can bind to the surface of heat shock protein homologues of $C$. albicans and lead to internalization and death of this organism [83]. The clinical signs of oral candidiasis have been investigated by counting Candida organisms in saliva [84], and a sensitive PCR detection system has been used to diagnose Pneumocystis carinii pneumonia [85].

Tables 1 and 2 presented the use of body fluid and diagnostic methods for the detection of opportunistic fungal infections in body fluids.

\section{Conclusions}

The examination of human body fluids is necessary to diagnose IFI, even though invasive methods may be needed to obtain samples for analysis. Routine laboratory tests for the diagnosis of FI include urinalysis and blood analysis. The second line for the diagnosis of systemic mycosis is the CSF. Analyses of both CSF and serum can improve the accuracy of the diagnosis. Other human body fluids should be obtained and analyzed for IFI according to specific conditions.

\section{Acknowledgments}

The author's special thanks go to Hassan Khajehei, Ph.D., for copy editing the paper and to K. Shashok (AuthorAID in the Eastern Mediterranean) for improving the use of English in the paper.

\section{References}

[1] C. Fourtounas, M. Marangos, P. Kalliakmani, E. Savidaki, D. S. Goumenos, and J. G. Vlachojannis, "Treatment of peritoneal dialysis related fungal peritonitis with caspofungin plus amphotericin B combination therapy," Nephrology Dialysis Transplantation, vol. 21, no. 1, pp. 236-237, 2006.

[2] P. Badiee and A. Alborzi, "Susceptibility of clinical Candida species isolates to antifungal agents by E-test, Southern Iran: a five year study," Iranian Journal of Microbiology, vol. 3, no. 4, pp. 183-188, 2011.

[3] S. C. Predari, A. N. De Paulis, D. Verón, A. Zucchini, and J. E. Santoianni, "Fungal peritonitis in patients on peritoneal dialysis: twenty five years of experience in a teaching hospital in Argentina," Revista Argentina de Microbiologia, vol. 39, no. 4, pp. 213-217, 2007.

[4] K. E. Black and L. R. Baden, "Fungal infections of the CNS: treatment strategies for the immunocompromised patient," CNS Drugs, vol. 21, no. 4, pp. 293-318, 2007.

[5] A. Chakrabarti, "Epidemiology of central nervous system mycoses," Neurology India, vol. 55, no. 3, pp. 191-197, 2007.

[6] J. M. K. Murthy, "Fungal infections of the central nervous system: the clinical syndromes," Neurology India, vol. 55, no. 3, pp. 221-225, 2007.

[7] P. Badiee, A. Alborzi, and M. Joukar, "Molecular assay to detect nosocomial fungal infections in intensive care units," European Journal of Internal Medicine, vol. 22, no. 6, pp. 611-615, 2011.

[8] C.-J. Huang, H. A. Pitt, P. A. Lipsett et al., "Pyogenic hepatic abscess: changing trends over 42 years," Annals of Surgery, vol. 223 , no. 5, pp. 600-609, 1996.

[9] G. J. Wise, G. S. Talluri, and V. K. Marella, "Fungal infections of the genitourinary system: manifestations, diagnosis, and treatment," Urologic Clinics of North America, vol. 26, no. 4, pp. 701-718, 1999.

[10] T. L. Pruett, O. D. Rotstein, J. Grass, M. P. Frick, A. Flohr, and R. L. Simmons, "Percutaneous aspiration and drainage for suspected abdominal infection," Surgery, vol. 96, no. 4, pp. 731737, 1984

[11] B. De Pauw, T. J. Walsh, J. P. Donnelly et al., "Revised definitions of invasive fungal disease from the European Organization for Research and Treatment of Cancer/Invasive Fungal Infections Cooperative Group and the National Institute of Allergy and Infectious Diseases Mycoses Study Group (EORTC/MSG) Consensus Group," Clinical Infectious Diseases, vol. 46, no. 12, pp. 1813-1821, 2008.

[12] S.-C. Ko, K.-Y. Chen, P.-R. Hsueh, K.-T. Luh, and P.-C. Yang, "Fungal empyema thoracis: an emerging clinical entity," Chest, vol. 117, no. 6, pp. 1672-1678, 2000.

[13] E. Hollenbach, "To treat or not to treat-critically ill patients with candiduria," Mycoses, vol. 51, no. 2, pp. 12-24, 2008.

[14] Z. A. Bukhary, "Candiduria: a review of clinical significance and management," Saudi Journal of Kidney Diseases and Transplantation, vol. 19, no. 3, pp. 350-360, 2008. 
[15] A. H. Limper, K. S. Knox, G. A. Sarosi et al., "An official American Thoracic Society statement: treatment of fungal infections in adult pulmonary and critical care patients," American Journal of Respiratory and Critical Care Medicine, vol. 183, no. 1, pp. 96128, 2011.

[16] P. Montravers, H. Dupont, R. Gauzit et al., "Candida as a risk factor for mortality in peritonitis," Critical Care Medicine, vol. 34, no. 3, pp. 646-652, 2006.

[17] P. Badiee, A. Alborzi, M. Moeini, and P. Haddadi, "Antifungal susceptibility of the Aspergillus species by Etest and CLSI Reference Methods," Archives of Iranian Medicine, vol. 15, no. 7, pp. 429-432, 2012.

[18] K.-Y. Chen, S.-C. Ko, P.-R. Hsueh, K.-T. Luh, and P.-C. Yang, "Pulmonary fungal infection: emphasis on microbiological spectra, patient outcome, and prognostic factors," Chest, vol. 120, no. 1, pp. 177-184, 2001.

[19] M. El-Ebiary, A. Torres, N. Fàbregas et al., "Significance of the isolation of Candida species from respiratory samples in critically ill, non-neutropenic patients: an immediate postmortem histologic study," American Journal of Respiratory and Critical Care Medicine, vol. 156, no. 2, pp. 583-590, 1997.

[20] M. Kimura, S.-I. Udagawa, K. Makimura, K. Satoh, N. Toyazaki, and H. Ito, "Isolation and identification of Rhizomucor pusillus from pleural zygomycosis in an immunocompetent patient," Medical Mycology, vol. 47, no. 8, pp. 869-873, 2009.

[21] L. S. Guazzelli, C. B. Severo, L. S. Hoff, G. L. F. Pinto, J. J. Camargo, and L. C. Severo, "Aspergillus fumigatus fungus ball in the pleural cavity," Jornal Brasileiro de Pneumologia, vol. 38, no. 1, pp. 125-132, 2012.

[22] M. A. Sackner, A. Wanner, and J. Landa, "Applications of bronchofiberoscopy," Chest, vol. 62, supplement 5, pp. 70-78, 1972.

[23] H. Y. Reynolds, "Immunologic system in the respiratory tract," Physiological Reviews, vol. 71, no. 4, pp. 1117-1133, 1991.

[24] J. Salonen, O.-P. Lehtonen, M.-R. Teräsjärvi, and J. Nikoskelainen, "Aspergillus antigen in serum, urine and bronchoalveolar lavage specimens of neutropenic patients in relation to clinical outcome," Scandinavian Journal of Infectious Diseases, vol. 32, no. 5, pp. 485-490, 2000.

[25] P. D. Khot, D. L. Ko, R. C. Hackman, and D. N. Fredricks, "Development and optimization of quantitative PCR for the diagnosis of invasive aspergillosis with bronchoalveolar lavage fluid," BMC Infectious Diseases, vol. 8, article 73, 2008.

[26] B. Musher, D. Fredricks, W. Leisenring, S. A. Balajee, C. Smith, and K. A. Marr, "Aspergillus galactomannan enzyme immunoassay and quantitative PCR for diagnosis of invasive aspergillosis with bronchoalveolar lavage fluid," Journal of Clinical Microbiology, vol. 42, no. 12, pp. 5517-5522, 2004.

[27] P. V. Murray, M. E. R. O’Brien, A. R. Padhani et al., "Use of first line bronchoalveolar lavage in the immunosuppressed oncology patient," Bone Marrow Transplantation, vol. 27, no. 9, pp. 967971, 2001.

[28] T. I. Chang, H. W. Kim, J. T. Park et al., "Early catheter removal improves patient survival in peritoneal dialysis patients with fungal peritonitis: results of ninety-four episodes of fungal peritonitis at a single center," Peritoneal Dialysis International, vol. 31, no. 1, pp. 60-66, 2011.

[29] P. G. Martos, F. G. de Sola, P. Marín et al., "Fungal peritonitis in continuous ambulatory peritoneal dialysis: a 10 cases description," Nefrologia, vol. 29, no. 6, pp. 534-539, 2009.

[30] P. Montravers, R. Gauzit, C. Muller, J. P. Marmuse, A. Fichelle, and J. M. Desmonts, "Emergence of antibiotic-resistant bacteria in cases of peritonitis after intraabdominal surgery affects the efficacy of empirical antimicrobial therapy," Clinical Infectious Diseases, vol. 23, no. 3, pp. 486-494, 1996.

[31] A. Roehrborn, L. Thomas, O. Potreck et al., "The microbiology of postoperative peritonitis," Clinical Infectious Diseases, vol. 33, no. 9, pp. 1513-1519, 2001.

[32] J. Matuszkiewicz-Rowinska, "Update on fungal peritonitis and its treatment," Peritoneal Dialysis International, vol. 29, supplement 2, pp. S161-S165, 2009.

[33] J. S. Solomkin, A. B. Flohr, P. G. Quie, and R. L. Simmons, “The role of Candida in intraperitoneal infections," Surgery, vol. 88, no. 4, pp. 524-530, 1980.

[34] H. Dupont, A. Bourichon, C. Paugam-Burtz, J. Mantz, and J.-M. Desmonts, "Can yeast isolation in peritoneal fluid be predicted in intensive care unit patients with peritonitis?" Critical Care Medicine, vol. 31, no. 3, pp. 752-757, 2003.

[35] Y.-S. Shan, H.-P. Hsu, Y.-H. Hsieh, E. D. Sy, J.-C. Lee, and P.-W. Lin, "Significance of intraoperative peritoneal culture of fungus in perforated peptic ulcer," British Journal of Surgery, vol. 90, no. 10, pp. 1215-1219, 2003.

[36] J. F. Fisher, J. D. Sobel, C. A. Kauffman, and C. A. Newman, "Candida urinary tract infections-treatment," Clinical Infectious Diseases, vol. 52, supplement 6, pp. S457-S466, 2011.

[37] C. A. Kauffman, J. A. Vazquez, J. D. Sobel et al., "Prospective multicenter surveillance study of funguria in hospitalized patients," Clinical Infectious Diseases, vol. 30, no. 1, pp. 14-18, 2000.

[38] O. Ayeni, K. M. Riederer, F. M. Wilson, and R. Khatib, "Clinicians' reaction to positive urine culture for Candida organisms," Mycoses, vol. 42, no. 4, pp. 285-289, 1999.

[39] S. P. Storfer, G. Medoff, V. J. Fraser, W. G. Powderly, and W. C. Dunagan, "Candiduria: retrospective review in hospitalized patients," Infectious Diseases in Clinical Practice, vol. 3, no. 1, pp. 23-29, 1994.

[40] J. D. Sobel, "Controversies in the diagnosis of candiduria: what is the critical colony count?" Current Treatment Options Infectious, vol. 4, pp. 81-83, 2002.

[41] E. D. Everett, T. C. Eickhofe, and J. M. Ehret, "Immunofluorescence of yeast in urine," Journal of Clinical Microbiology, vol. 2, no. 2, pp. 142-143, 1975.

[42] J. Schonebeck, "Studies on Candida infection of the urinary tract and on the antimycotic drug 5-fluorocytosine," Scandinavian Journal of Urology and Nephrology, vol. 1, supplement 11, pp. 7-48, 1972.

[43] E. E. Navarro, J. S. Almario, C. King, J. Bacher, P. A. Pizzo, and T. J. Walsh, "Detection of Candida casts in experimental renal candidiasis: implications for the diagnosis and pathogenesis of upper urinary tract infection," Journal of Medical and Veterinary Mycology, vol. 32, no. 6, pp. 415-426, 1994.

[44] C. Argyle, G. B. Schumann, L. Genack, and M. Gregory, "Identification of fungal casts in a patient with renal candidiasis," Human Pathology, vol. 15, no. 5, pp. 480-481, 1984.

[45] S. A. Harding and W. G. Merz, "Evaluation of antibody coating of yeasts in urine as an indicator of the site of urinary tract infection," Journal of Clinical Microbiology, vol. 2, no. 3, pp. 222225, 1975.

[46] W. J. Hall, "Study of antibody-coated fungi in patients with funguria and suspected disseminated fungal infections or primary fungal pyelonephritis," Journal of the Royal Society of Medicine, vol. 73, no. 8, pp. 567-569, 1980. 
[47] J. F. Fisher, K. Kavanagh, J. D. Sobel, C. A. Kauffman, and C. A. Newman, "Candida urinary tract infection: pathogenesis," Clinical Infectious Diseases, vol. 52, supplement 6, pp. S437-S451, 2011.

[48] C. A. Kauffman, "Candiduria: diagnostic and treatment conundrums," Current Treatment Options Infectious Diseases, vol. 4, pp. 513-519, 2002.

[49] E. E. Navarro, J. S. Almario, R. L. Schaufele, J. Bacher, and T. J. Walsh, "Quantitative urine cultures do not reliably detect renal candidiasis in rabbits," Journal of Clinical Microbiology, vol. 35, no. 12, pp. 3292-3297, 1997.

[50] C. C. Canver, A. K. Patel, P. Kosolcharoen, and M. C. Voytovich, "Fungal purulent constrictive pericarditis in a heart transplant patient," Annals of Thoracic Surgery, vol. 65, no. 6, pp. 1792-1794, 1998.

[51] R. Rabinovici, D. Szewczyk, P. Ovadia, J. R. Greenspan, and J. J. Sivalingam, "Candida pericarditis: clinical profile and treatment," Annals of Thoracic Surgery, vol. 63, no. 4, pp. 12001204, 1997.

[52] M. B. Cishek, B. Yost, and S. Schaefer, "Cardiac aspergillosis presenting as myocardial infarction," Clinical Cardiology, vol. 19, no. 10 , pp. $824-827,1996$.

[53] J. Wheat, "Histoplasmosis: experience during outbreaks in Indianapolis and review of the literature," Medicine, vol. 76, no. 5, pp. 339-354, 1997.

[54] D. H. Spodick, The Pericardium: A Comprehensive Textbook, Marcel Dekker, New York, NY, USA, 1997.

[55] B. Maisch, P. M. Seferović, A. D. Ristić et al., "Guidelines on the diagnosis and management of pericardial diseases executive summary the task force on the diagnosis and management of pericardial diseases of the European society of cardiology," European Heart Journal, vol. 25, no. 7, pp. 587-610, 2004.

[56] P. Badiee, A. Alborzi, E. Shakiba, M. Ziyaeyan, and B. Pourabbas, "Molecular diagnosis of Aspergillus endocarditis after cardiac surgery," Journal of Medical Microbiology, vol. 58, no. 2, pp. 192-195, 2009.

[57] A. R. Kosmin and T. Fekete, "Use of fungal blood cultures in an Academic Medical Center," Journal of Clinical Microbiology, vol. 46, no. 11, pp. 3800-3801, 2008.

[58] D. A. Stevens, V. L. Kan, M. A. Judson et al., "Practice guidelines for diseases caused by Aspergillus," Clinical Infectious Diseases, vol. 30, no. 4, pp. 696-709, 2000.

[59] J. C. Varghese, P. F. Hahn, M. G. Harisinghani et al., "Fungusinfected fluid collections in thorax or abdomen: effectiveness of percutaneous catheter drainage," Radiology, vol. 236, no. 2, pp. 730-738, 2005.

[60] M. E. Falagas, K. E. Apostolou, and V. D. Pappas, "Attributable mortality of candidemia: a systematic review of matched cohort and case-control studies," European Journal of Clinical Microbiology \& Infectious Diseases, vol. 25, no. 7, pp. 419-425, 2006.

[61] J. W. Pickering, H. W. Sant, C. A. P. Bowles, W. L. Roberts, and G. L. Woods, "Evaluation of a $(1 \rightarrow 3)-\beta$-D-glucan assay for diagnosis of invasive fungal infections," Journal of Clinical Microbiology, vol. 43, no. 12, pp. 5957-5962, 2005.

[62] L. Ostrosky-Zeichner, B. D. Alexander, D. H. Kett et al., "Multicenter clinical evaluation of the $(1 \rightarrow 3) \beta$-D-glucan assay as an aid to diagnosis of fungal infections in humans," Clinical Infectious Diseases, vol. 41, no. 5, pp. 654-659, 2005.

[63] C. Viscoli, M. Machetti, P. Gazzola et al., "Aspergillus galactomannan antigen in the cerebrospinal fluid of bone marrow transplant recipients with probable cerebral aspergillosis," Journal of Clinical Microbiology, vol. 40, no. 4, pp. 1496-1499, 2002.
[64] D. Rimek, K. Redetzke, J. Singh, K. Heinrich, and R. Kappe, "Performance of the Candida mannan antigen detection in patients with fungemia," Mycoses, vol. 47, no. 1, pp. 23-26, 2004.

[65] H. Yera, B. Sendid, N. Francois, D. Camus, and D. Poulain, "Contribution of serological tests and blood culture to the early diagnosis of systemic candidiasis," European Journal of Clinical Microbiology \& Infectious Diseases, vol. 20, no. 12, pp. 864-870, 2001.

[66] P. Badiee, P. Kordbacheh, A. Alborzi, and S. A. Malekhoseini, "Invasive fungal infection in renal transplant recipients demonstrated by panfungal polymerase chain reaction," Experimental and Clinical Transplantation, vol. 5, no. 1, pp. 624-629, 2007.

[67] C. Lass-Flörl, E. Gunsilius, G. Gastl et al., "Diagnosing invasive aspergillosis during antifungal therapy by PCR analysis of blood samples," Journal of Clinical Microbiology, vol. 42, no. 9, pp. 4154-4157, 2004.

[68] A. J. M. Van Deventer, W. H. F. Goessens, A. Van Belkum, E. W. M. Van Etten, H. J. A. Van Vliet, and H. A. Verbrugh, "PCR monitoring of response to liposomal amphotericin B treatment of systemic candidiasis in neutropenic mice," Journal of Clinical Microbiology, vol. 34, no. 1, pp. 25-28, 1996.

[69] P. Badiee, P. Kordbacheh, A. Alborzi et al., "Study on invasive fungal infections in immunocompromised patients to present a suitable early diagnostic procedure," International Journal of Infectious Diseases, vol. 13, no. 1, pp. 97-102, 2009.

[70] P. Badiee and A. Alborzi, "Detection of Aspergillus species in bone marrow transplant patients," Journal of Infection in Developing Countries, vol. 4, no. 8, pp. 511-516, 2010.

[71] P. Badiee, A. Alborzi, R. Vojdani et al., "Early diagnosis of systemic candidiasis in bone marrow transplant recipients," Experimental and Clinical Transplantation, vol. 8, no. 2, pp. 98103, 2010.

[72] A. C. Pettit, J. A. Kropski, J. L. Castilho et al., "The index case for the fungal meningitis outbreak in the United States," The New England Journal of Medicine, vol. 367, no. 22, pp. 2119-2125, 2012.

[73] D. L. Pagano, C. Girmenia, L. Mele et al., "Infections caused by filamentous fungi in patients with hematologic malignancies. A report of 391 cases by GINEMA infection program," Haematologica, vol. 86, no. 8, pp. 862-870, 2001.

[74] D. A. Jerrard, J. R. Hanna, and G. L. Schindelheim, "Cerebrospinal fluid," Journal of Emergency Medicine, vol. 21, no. 2, pp. 171-178, 2001.

[75] J. Davis, D. Costello, and N. Venna, "Laboratory investigation of fungal infections of the central nervous system," Neurology India, vol. 55, no. 3, pp. 233-240, 2007.

[76] C. S. F. Purulent, "Cerebrospinal fluid analysis", American Familiar Physician, vol. 68, no. 6, pp. 1103-1109, 2003.

[77] R. K. Khurana, "Intracranial Hypotension," Seminars in Neurology, vol. 16, no. 1, pp. 5-10, 1996.

[78] P. Badiee and A. Alborzi, "Assessment of a real-time PCR method to detect human non-cryptococcal fungal meningitis," Archives of Iranian Medicine, vol. 14, no. 6, pp. 381-384, 2011.

[79] R. Golmia, I. Bello, A. Marra, N. Hamerschlak, A. Osawa, and M. Scheinberg, "Aspergillus fumigatus joint infection: a review," Seminars in Arthritis and Rheumatism, vol. 40, no. 6, pp. 580584, 2011.

[80] B. H. Hwang, J. Y. Yoon, C. H. Nam et al., "Fungal periprosthetic joint infection after primary total knee replacement," British Journal of Bone \& Joint Surgery, vol. 94, no. 5, pp. 656659, 2012. 
[81] J. Merrer, B. Dupont, A. Nieszkowska, B. De Jonghe, and H. Outin, "Candida albicans prosthetic arthritis treated with fluconazole alone," Journal of Infection, vol. 42, no. 3, pp. 208209, 2001.

[82] T. K. Fábián, P. Hermann, A. Beck, P. Fejérdy, and G. Fábián, "Salivary defense proteins: their network and role in innate and acquired oral immunity," International Journal of Molecular Sciences, vol. 13, no. 4, pp. 4295-4320, 2012.

[83] S. R. Torres, A. Garzino-Demo, T. F. Meiller, V. Meeks, and M. A. Jabra-Rizk, "Salivary histatin-5 and oral fungal colonisation in HIV+ individuals," Mycoses, vol. 52, no. 1, pp. 11-15, 2009.

[84] M. Navazesh, G. J. Wood, and V. J. Brightman, "Relationship between salivary flow rates and Candida albicans counts," Oral Surgery, Oral Medicine, Oral Pathology, Oral Radiology and Endodontology, vol. 80, no. 3, pp. 284-288, 1995.

[85] S. Fischer, V. J. Gill, J. Kovacs et al., "The use of oral washes to diagnose Pneumocystis carinii pneumonia: a blinded prospective study using a polymerase chain reaction-based detection system," The Journal of Infectious Diseases, vol. 184, no. 11, pp. 1485-1488, 2001. 

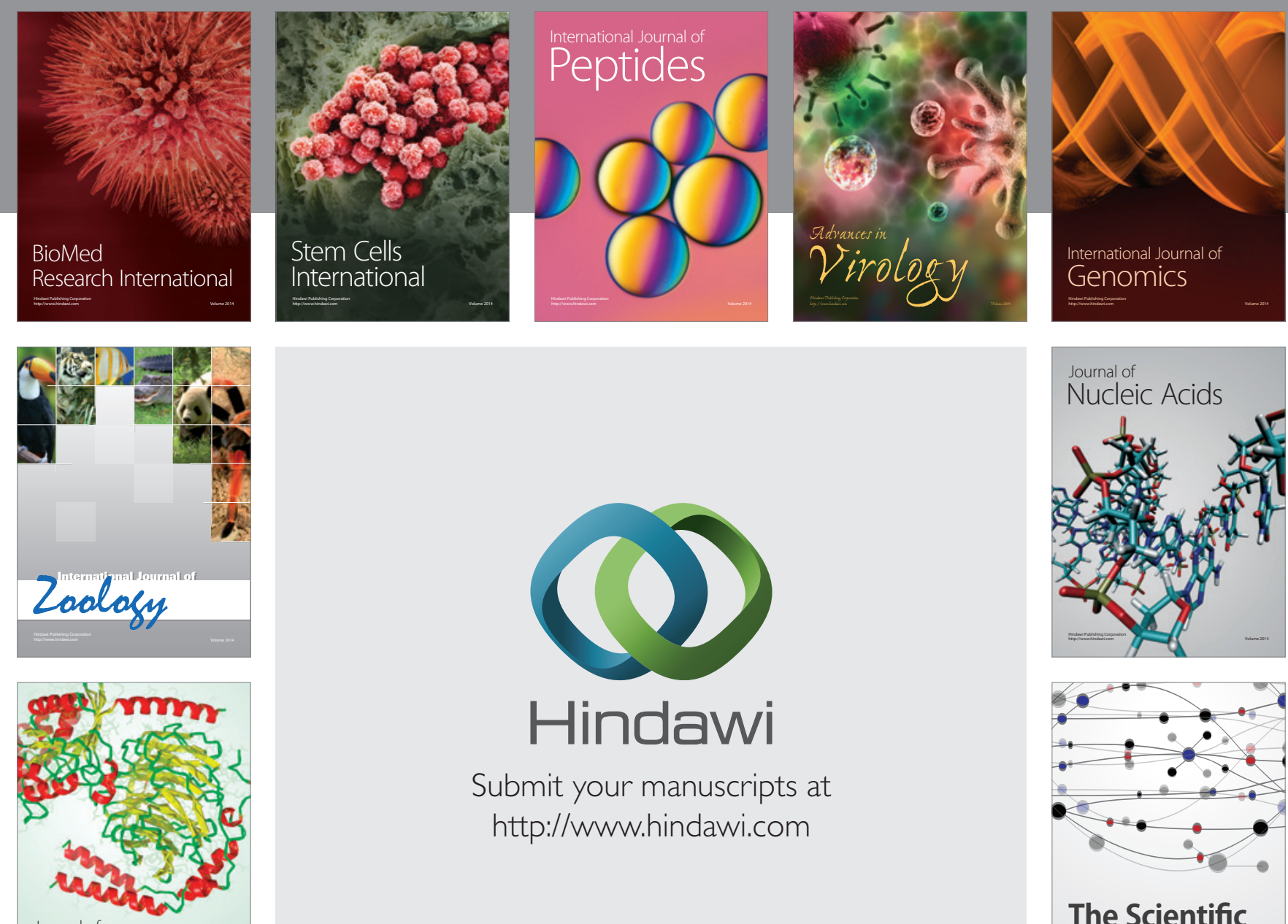

Submit your manuscripts at

http://www.hindawi.com

Journal of
Signal Transduction
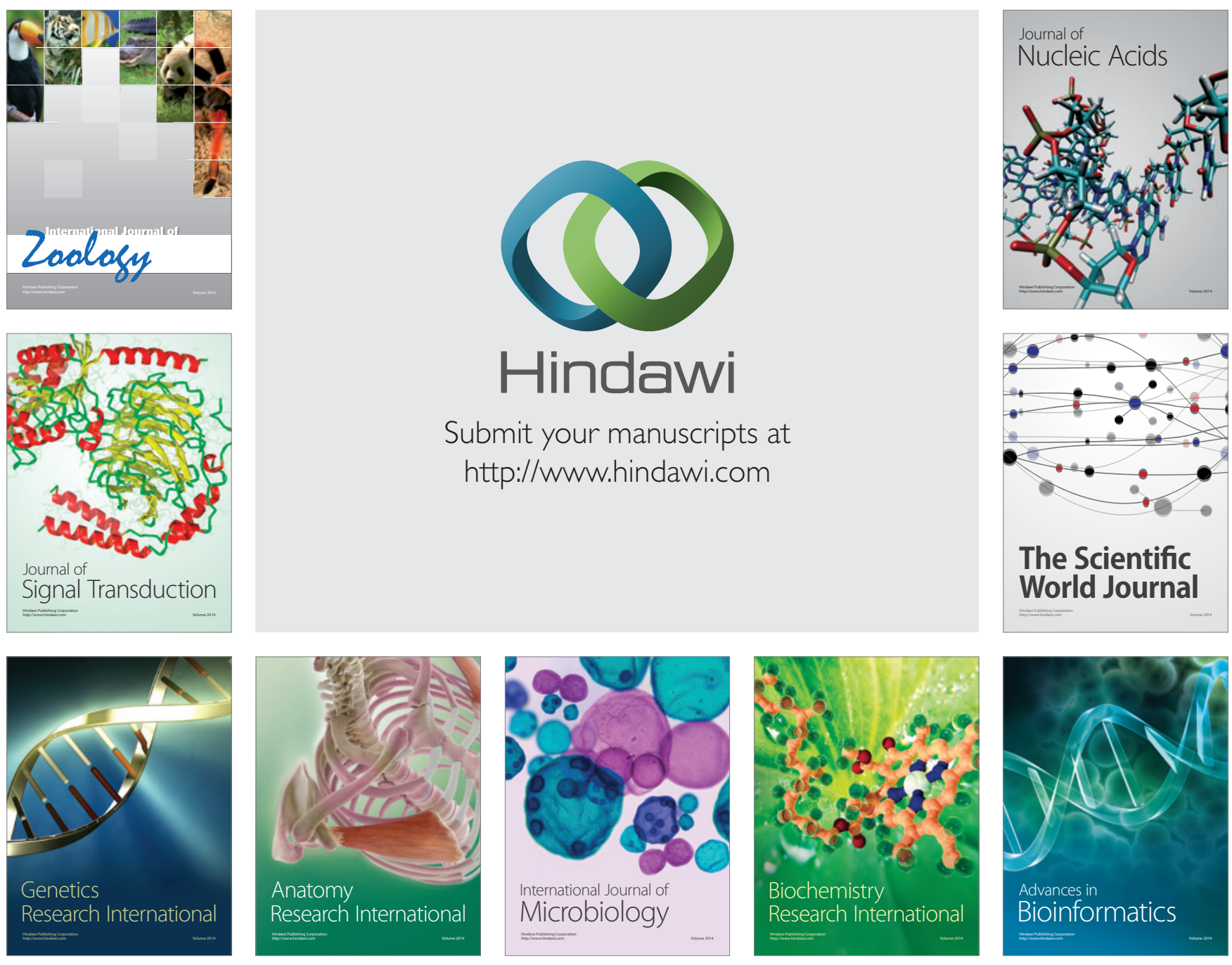

The Scientific World Journal
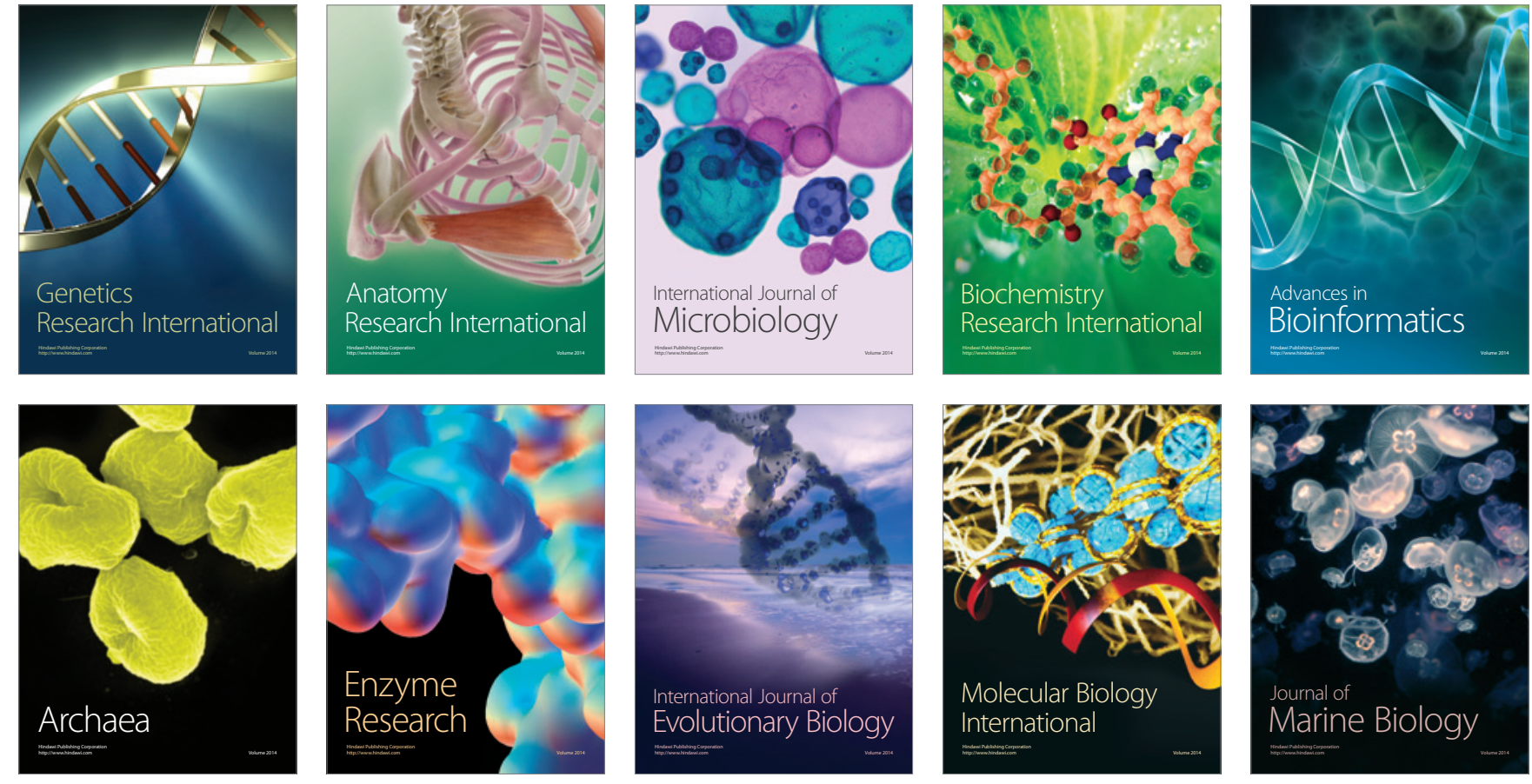